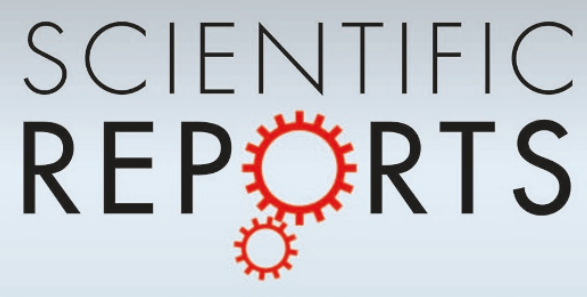

OPEN

SUBJECT AREAS:

POROUS MATERIALS

POLYMERS

CONJUGATED POLYMERS

MAGNETIC PROPERTIES AND

MATERIALS

Received

23 April 2013

Accepted

17 June 2013

Published

3 July 2013

Correspondence and requests for materials should be addressed to

B.T. (bien.tan@mail. hust.edu.cn)

* These authors contributed equally to this work.

\section{Hollow Microporous Organic Capsules}

\author{
Buyi Li1*, Xinjia Yang ${ }^{1 *}$, Lingling Xia' ${ }^{1}$, Muhammad Irfan Majeed' \& Bien Tan ${ }^{1,2,3}$
}

'School of Chemistry and Chemical Engineering, Huazhong University of Science and Technology, Luoyu Road No. 1037, Wuhan, 430074, China, ${ }^{2}$ Hubei Key Laboratory of Material Chemistry and Service Failure, Huazhong University of Science and Technology, Luoyu Road No. 1037, Wuhan, 430074, China, ${ }^{3}$ Key Laboratory for Large-Format Battery Materials and System, Ministry of Education Huazhong University of Science and Technology, Luoyu Road No. 1037, Wuhan, 430074, China.

Fabrication of hollow microporous organic capsules (HMOCs) could be very useful because of their hollow and porous morphology, which combines the advantages of both microporous organic polymers and non-porous nanocapsules. They can be used as storage materials or reaction chambers while supplying the necessary path for the design of controlled uptake/release systems. Herein, the synthesis of HMOCs with high surface area through facile emulsion polymerization and hypercrosslinking reactions, is described. Due to their tailored porous structure, these capsules possessed high drug loading efficiency, zero-order drug release kinetics and are also demonstrated to be used as nanoscale reactors for the prepareation of nanoparticles (NPs) without any external stabilizer. Moreover, owing to their intrinsic biocompatibility and fluorescence, these capsules exhibit promising prospect for biomedical applications. 


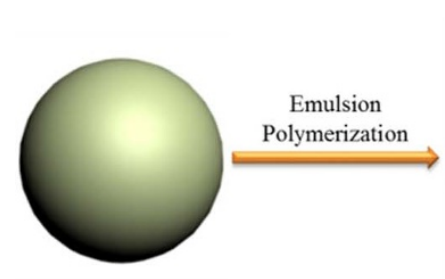

$\mathrm{SiO}_{2} \mathrm{NPS}$

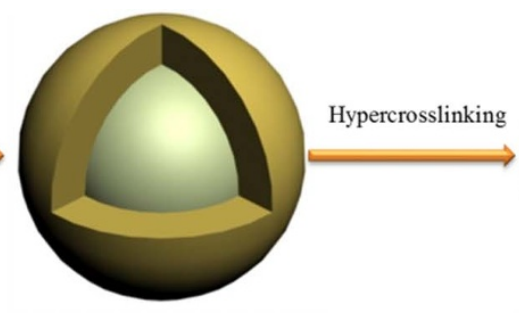

$\mathrm{SiO}_{2} @$ PS-DVB

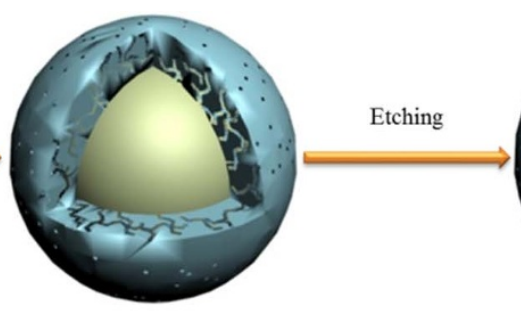

Hypercrosslinked $\mathrm{SiO}_{2} @$ PS-DVB

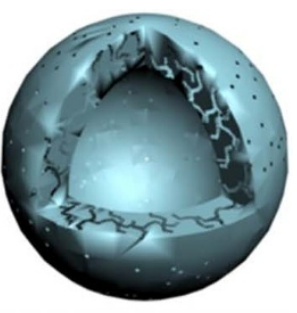

Hollow Microporous Organic Capsules (HMOCs)

Figure 1 Schematic synthetic route of hollow microporous organic capsules (HMOCs).

\section{Results}

$\mathrm{SiO}_{2} @ P S-D V B$ precursor nanoparticles. Silica nanoparticles $\left(\mathrm{SiO}_{2}\right.$ NPs) were synthesized through traditional Stöber method. The vinyl group of 3-(trimethoxysilyl)propyl methacrylate (MPS) grafted on the $\mathrm{SiO}_{2} \mathrm{NPs}$ can react with monomers (styrene and DVB) to form macromolecular chains anchored on their surface, thus forming $\mathrm{SiO}_{2} @ P S-D V B$ core-shell structures ${ }^{43}$. Previously, we have demonstrated that the pore structure can be controlled from macro to microscale by changing the DVB contents ${ }^{44}$. Hence, to vary the porous structure of HMOCs, a series of precursor NPs $\left(\mathrm{SiO}_{2} @ P S-\right.$ DVB) were prepared with different DVB contents $(0.5,1,2.5,5,10$ and $15 \mathrm{wt} . \%$ of styrene). It was observed that co-monomer (DVB) has a significant effect on the core-shell morphology of $\mathrm{SiO}_{2} @ P S-D V B$ NPs (Fig. 2). The $\mathrm{SiO}_{2} @ P S-D V B$ NPs with 0.5, 1 and 2.5\% DVB exhibited eccentric $\mathrm{SiO}_{2}$ cores, while the $\mathrm{SiO}_{2}$ cores in $\mathrm{SiO}_{2} @ P S-$ DVB NPs with 5, 10 and 15\% DVB were centric. By increasing the DVB contents, the $\mathrm{SiO}_{2} @ P S-D V B$ tended to have concentric core shell morphology. By decreasing the DVB contents were low, the PS chains were weakly crosslinked by DVB and the rigidity of the shell could not be balanced with the gravity of $\mathrm{SiO}_{2}$ core. Increasing the DVB content enhanced the rigidity of the shell, therefore the $\mathrm{SiO}_{2}$ spheres tended to be located in the center ${ }^{44}$.

Hypercrosslinking reactions and hollow microporous organic capsules. After hypercrosslinking, sacrificial $\mathrm{SiO}_{2}$ cores were etched by hydrofluoric acid (HF). From TEM and SEM images of precursor ( $\left.\mathrm{SiO}_{2} @ P S-D V B\right)$ NPs (Fig. 2 and Fig. S1-S6) and hollow HMOCs (Fig. 3a to 3 f and Fig. S7-S12) after hypercrosslinking and etching,it is obvious that HMOCs retained their original morphology

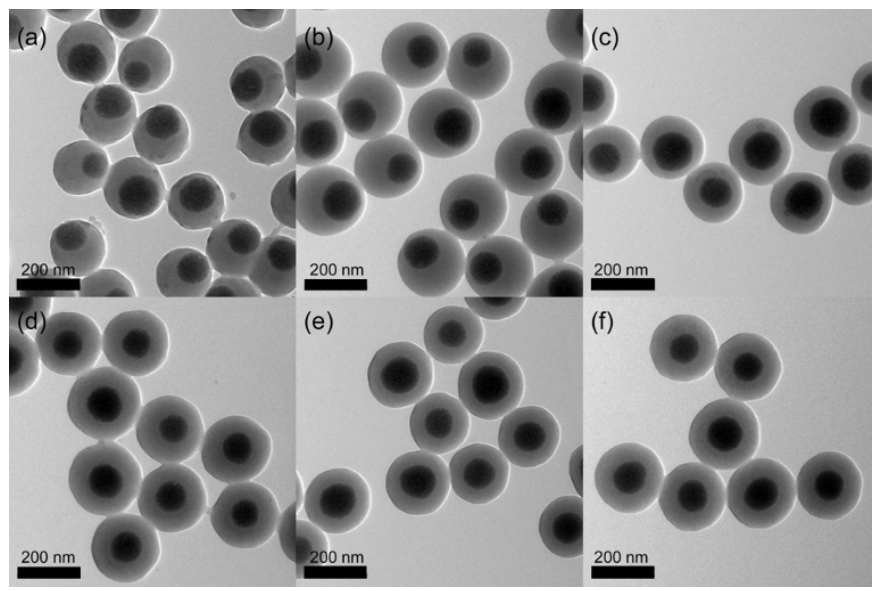

Figure 2 TEM images of $\mathrm{SiO}_{2} @ P S$ core-shell precursors with different DVB contents. (a) $\mathrm{SiO}_{2} @ P S-0.5 \%$ DVB, (b) $\mathrm{SiO}_{2} @ P S-1 \%$ DVB, (c) $\mathrm{SiO}_{2} @$ PS-2.5\% DVB, (d) $\mathrm{SiO}_{2} @ P S-5 \%$ DVB, (e) $\mathrm{SiO}_{2} @ P S-10 \%$ DVB, (f) $\mathrm{SiO}_{2} @$ PS-15\% DVB. Styrene is $10 \mathrm{ml}$. The $\mathrm{SiO}_{2}$ nanoparticles core is $130 \mathrm{~nm}$. The mass of $\mathrm{SiO}_{2}$ nanoparticles is $1.2 \mathrm{~g}$. The scale is $200 \mathrm{~nm}$. after the removal of $\mathrm{SiO}_{2}$ cores. Low DVB contents (0.5 wt.\%) led to the formation of HMOCs with rough surface. This might be due the low degree of crosslinking resulting in the twisting of some macromolecular chains leading to the rough surface. Shell's surface became smooth when the DVB content was up to $1 \mathrm{wt}$ \% or higher. These observations are in consistence with the previous reports ${ }^{44}$. The HMOCs with varying size of hollow cavities and different
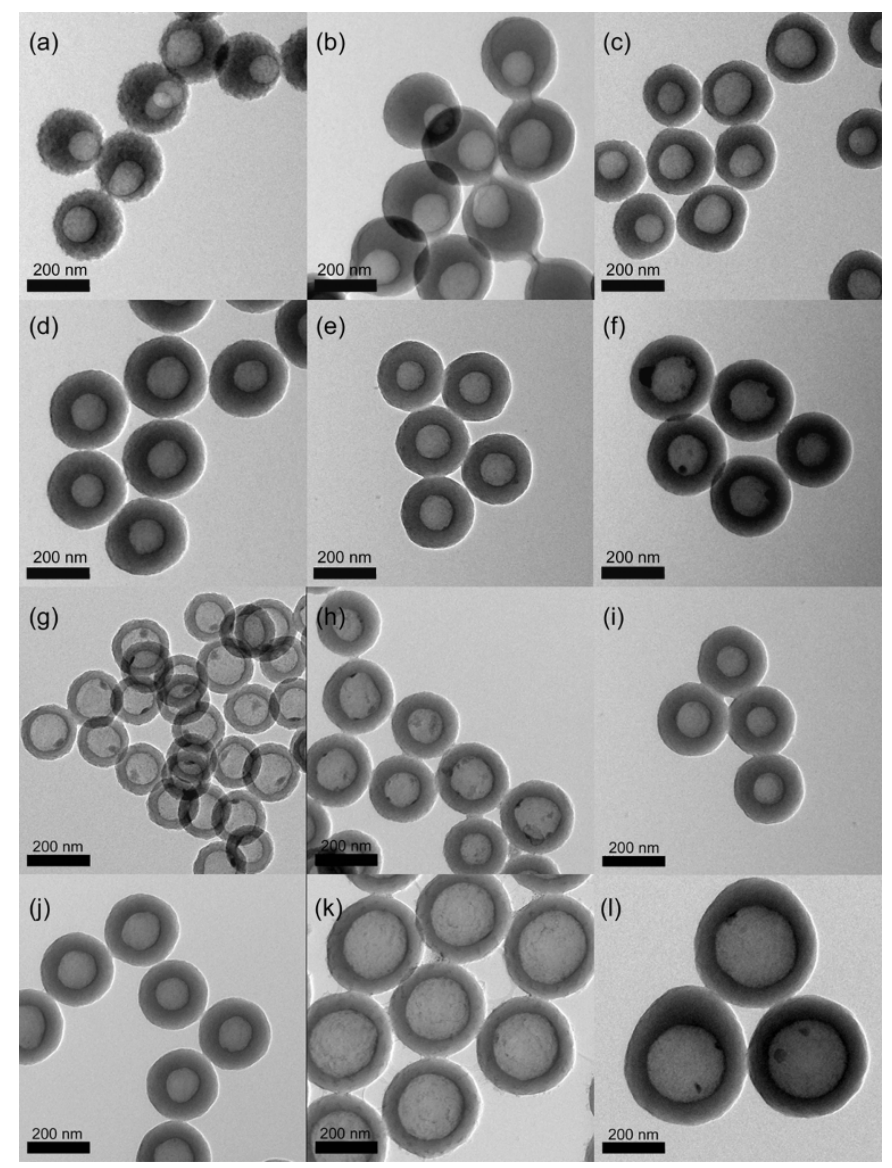

(i)

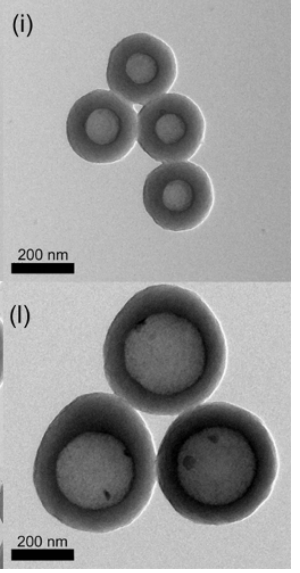

Figure 3 TEM images of HMOCs obtained after hypercrosslinking of $\mathrm{SiO}_{2} @ \mathrm{PS}$ core-shell precursors with different DVB content and etching $\mathrm{SiO}_{2}$ core. (a) $0.5 \%$ - HMOCs, (b) $1 \%$ - HMOCs, (c) $2.5 \%$ - HMOCs, (d) $5 \%$ - HMOCs, (e) $10 \%$ - HMOCs, (f) $15 \%$ - HMOCs. Styrene is $10 \mathrm{ml}$ with $130 \mathrm{~nm}$ hollow cavities. TEM images of HMOCs with different thickness of the shell by varying the dose of styrene in $\mathrm{SiO}_{2} @ \mathrm{PS}$ core-shell precursors, (g) $10 \%$ - HMOCs - $2.5 \mathrm{ml}$, (h) 10\% - HMOCs - $5 \mathrm{ml}$, (i) 10\% - HMOCs $10 \mathrm{ml}$, (j) 10\% - HMOCs - $15 \mathrm{ml}$ with $130 \mathrm{~nm}$ hollow cavities. TEM image of HMOCs with $200 \mathrm{~nm}$ hollow cavities. (k) 10\% - HMOCs - $5 \mathrm{ml}-$ $200 \mathrm{~nm}$, (1) $10 \%$ - HMOCs - $10 \mathrm{ml}-200 \mathrm{~nm}$. The mass of $\mathrm{SiO}_{2}$ nanoparticles is $1.2 \mathrm{~g}$. The scale is $200 \mathrm{~nm}$. 
shell thickness were obtained as expected. In order to obtain the HMOCs with different shell thickness, the dose of styrene was varied from 2.5 to $15 \mathrm{ml}$ for the synthesis of $\mathrm{SiO}_{2} @ P S-D V B$ nanoparticles with $\sim 130 \mathrm{~nm} \mathrm{SiO}$ cores (S15-S18). The TEM images of HMOCs after hypercrosslinking and etching are shown in Fig. $\mathbf{3 g}$ to $3 j$. It was observed that the resulting particle size (including core and shell) and shell thickness (excluding core) increased with a decrease in initial emulsifier to monomer ratio which was consistent with the dominant micellar nucleation mechanism proposed by Harkins ${ }^{45}$. According to this mechanism, a decrease in the initial emulsifier to monomer ratio decreases the relative amount of micelles which in turn decreases the number of polymer particle nuclei formed early in the reaction per unit time resulting in formation of polymer particles with smaller in numbers but larger in size ${ }^{4}$. It was also possible to change the size of hollow cavities of HMOCs (Fig. 3k and 3l) by using $\mathrm{SiO}_{2}$ cores with different sizes (e.g. $200 \mathrm{~nm}$, Fig. S19 and S20). The mass of $\mathrm{SiO}_{2}$ nanoparticles is $1.2 \mathrm{~g}$.

Surface areas and porous structures. It is clearly evident from the TEM images that the size of hollow cavity of HMOCs and their shell thickness can easily be tuned. However, the microporous structure of their shell cannot be observed by these imaging techniques. Nitrogen gas adsorption and desorption experiment were, therefore, employed to confirm their microporous structure. The BET surface area, Langmuir surface area, and pore volume are summarized in Table 1 and Table S1. It is clearly evident that by increasing the DVB contents, the surface area of HMOCs decreases, which can be attributed to the decrease in the hypercrosslinking of styrene. The nitrogen adsorption and desorption isotherms were obtained at 77.3 K (Fig. 4a) and pore size distribution of HMOCs with various DVB contents was calculated using DFT methods (slit pore models, differential pore volume vs pore width, Fig. 4b). As shown in Fig. 4a, the nitrogen adsorption isotherms of all HMOCs exhibit a steep rise at low relative pressure $\left(\mathrm{P} / \mathrm{P}_{0}<0.001\right)$, reflecting the abundance of microporous structure, while a steep rise at high pressure $\left(\mathrm{P} / \mathrm{P}_{0}=1\right)$ may be due to the macropores formed as interstitial voids between the highly aggregated capsules and their hollow interior ${ }^{46}$. Nitrogen adsorption and desorption isotherms of HMOCs with $0.5 \%$ to $5 \%$ DVB have clear hysteresis loop which indicates the mesoporous structure of capsules shell ${ }^{46}$. The pore size distribution (Fig. 4b) also shows the presence of micropores, mesopores and macropores in the materials simultaneously. With increasing the DVB contents to 10 and $15 \%$, the hysteresis loop disappears and becomes flat in the intermediate section, which suggests that the mesoporous structure is diminished with an increase in DVB contents. Since, there are no peaks in the mesoporous region of pore size distribution curves, it indicates a sheer microporous structure of the capsule shell ${ }^{46}$. With an increase in DVB contents, in addition to the disappearance of mesoporous structure, smaller micropores are also formed as it is evident from obvious shift of the peaks to the lower value in pore size (a)

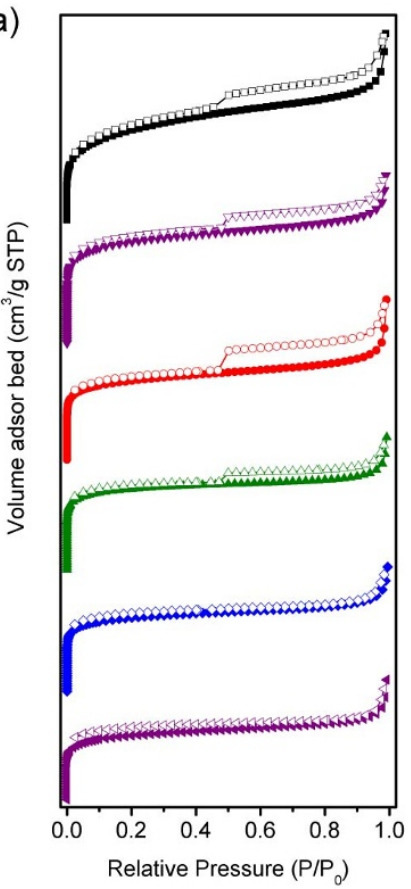

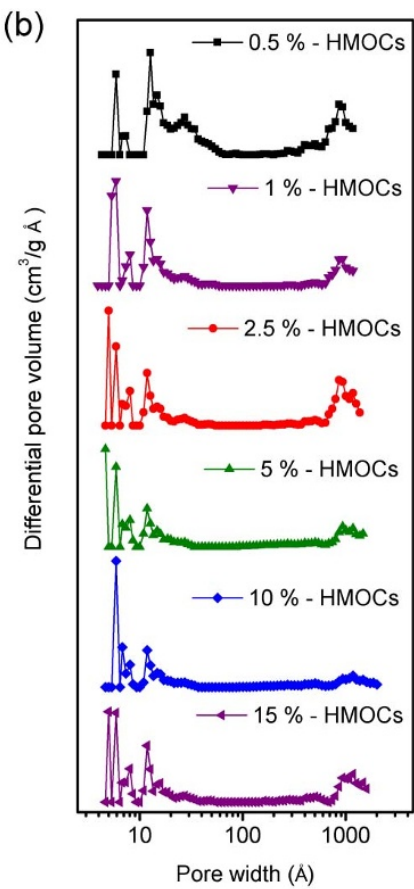

Figure $4 \mid$ (a) Nitrogen sorption isotherms at $77.3 \mathrm{~K}$ and (b) pore distribution of pore size calculated using DFT methods (slit pore models, differential pore volumes) of HMOCs with $130 \mathrm{~nm}$ hollow cavity.

distribution curves in the region below $2 \mathrm{~nm}$ (Fig. 4b). As shown in Table 1, pore volume of HMOCs reduces gradually with an increase of DVB content, which also demonstrates the transformation of the mesopores to the micropores.

\section{Discussion}

HMOCs were also evaluated for their possible potential biomedical applications. Prior to that, the toxicity of HMOCs was investigated through an MTT assay, and cell viability of HepG2 cells was determined in the presence of HMOCs (Fig. S21). HMOCs showed almost no cytotoxicity over the range of concentrations studied $(0,15.6$, $62.5,125,250$ and $500 \mu \mathrm{g} / \mathrm{mL}$ ). As HMOCs are around $250 \mathrm{~nm}$ in diameter, usually they will be sequestered by phagocytotic cells of the spleen and eliminated from the body eventually ${ }^{47}$. Interestingly, these HMOCs were also found to be fluorescent, which may be useful for their applications in bio-imaging and bio-labeling. The origin of fluorescence in these HMOCs is not clear yet but it may be due the stacking of benzene rings and nanoscale size effect. The optical images of HMOCs under UV light in simulated body fluid (PBS, $\mathrm{pH}=7.4$, buffer solution) are shown in Fig. S22. The emission

Table 1 | Surface area and porosity of HMOCs

\begin{tabular}{|c|c|c|c|c|c|c|c|}
\hline Samples & DVB \% & $\mathrm{S}_{\mathrm{BET}}{ }^{[\mathrm{a]}]} \mathrm{m}^{2} / \mathrm{g}$ & $\mathrm{S}_{\mathrm{L}}^{[\mathrm{b}]} \mathrm{m}^{2} / \mathrm{g}$ & M.A. ${ }^{[c]} \mathrm{m}^{2} / \mathrm{g}$ & $P V^{[d]} \mathrm{cm}^{3} / \mathrm{g}$ & M.P.V. ${ }^{[\mathrm{e}]} \mathrm{cm}^{3} / \mathrm{g}$ & M.A. ${ }^{[f]} \%$ \\
\hline $0.5 \%-\mathrm{HMOCs}$ & 0.5 & 1129 & 1549 & 361 & 0.98 & 0.15 & 32.0 \\
\hline $1 \%-\mathrm{HMOCs}$ & 1 & 815 & 1098 & 496 & 0.61 & 0.22 & 60.8 \\
\hline $2.5 \%-\mathrm{HMOCs}$ & 2.5 & 697 & 932 & 431 & 0.59 & 0.20 & 61.8 \\
\hline $10 \%-\mathrm{HMOCs}$ & 10 & 516 & 691 & 351 & 0.35 & 0.16 & 68.0 \\
\hline $15 \%-\mathrm{HMOCs}$ & 15 & 478 & 640 & 296 & 0.35 & 0.14 & 61.9 \\
\hline 10\% - Solid HCPs & 10 & 616 & 829 & - & 0.83 & - & - \\
\hline
\end{tabular}

a] Surface area calculated from nitrogen adsorption isotherms at $77.3 \mathrm{~K}$ using BET equation.

[b] Surface area calculated from nitrogen adsorption isotherms at $77.3 \mathrm{~K}$ using Langmuir equation.

${ }^{[\mathrm{c}]} \mathrm{t}$-Plot micropore area.

[d]Pore volume calculated from nitrogen isotherm at $\mathrm{P} / \mathrm{P}_{0}=0.995,77.3 \mathrm{~K}$.

[e] + -Plot micropore volume.

$\left[f_{t}\right.$-Plot micropore area. /BET surface area*100\%. 
spectra of HMOCs (Fig. S23-S28) indicate the emission peak at $590 \mathrm{~nm}$ when excited at $440 \mathrm{~nm}$. In order to evaluate their application in a drug delivery system, in vitro drug loading and release experiments were performed with HMOCs having different pore structures using ibuprofen (IBU) as a model drug. Loading of the drug was achieved in IBU hexane solution, and the drug release rate was obtained by soaking the drug-loaded HMOCs in simulated body fluid (PBS, $\mathrm{pH}=7.4$, buffer solution). The drug loading efficiency of HMOCs was calculated from the data obtained with UV-vis spectrophotometric and thermogravimetric (TG) analysis. In Fig. S29-S39, the weight loss at and above $450^{\circ} \mathrm{C}$ corresponds to the decomposition of HMOCs itself. But prior to that, the weight loss observed in the range of $180-230^{\circ} \mathrm{C}$ can be attributed due to the removal of IBU. As summarized in Table S2, HMOCs can uptake 1.68 to $2.04 \mathrm{~g}$ of ibuprofen $/ \mathrm{g}$, which is much higher than that $(0.80 \mathrm{~g}$ of ibuprofen $/ \mathrm{g})$ achieved for solid HCP nanoparticles prepared in the absence of silica core via similar synthesis process. The $10 \%$-solid HCPs possess higher surface area $\left(616 \mathrm{~m}^{2} / \mathrm{g}\right)$ than that $\left(516 \mathrm{~m}^{2} / \mathrm{g}\right)$ of $10 \%$ HMOCs, but much lower drug uptake $(0.84 \mathrm{~g}$ of ibuprofen $/ \mathrm{g})$ than that of $10 \%$ - HMOCs (1.85 g of ibuprofen/g), which indicates the entrapment of drug inside the hollow cavity of HMOCs. The surface area of $10 \%$ - HMOCs was found to be lower than that of $10 \%$ - solid HCPs, which may be due to an extra internal surface of $10 \%$ HMOCs for being hollow. Moreover, the benzene rings present on the external and internal surfaces of precursor shell cannot been hypercrosslinked as effectively as those present inside the precursor shell ${ }^{4}$. The drug release profile (Fig. 5) indicates that the porous structure of shell also affects the drug release kinetics. The shell of $0.5 \%$ - HMOCs to $5 \%$ - HMOCs possess meso- and microporous

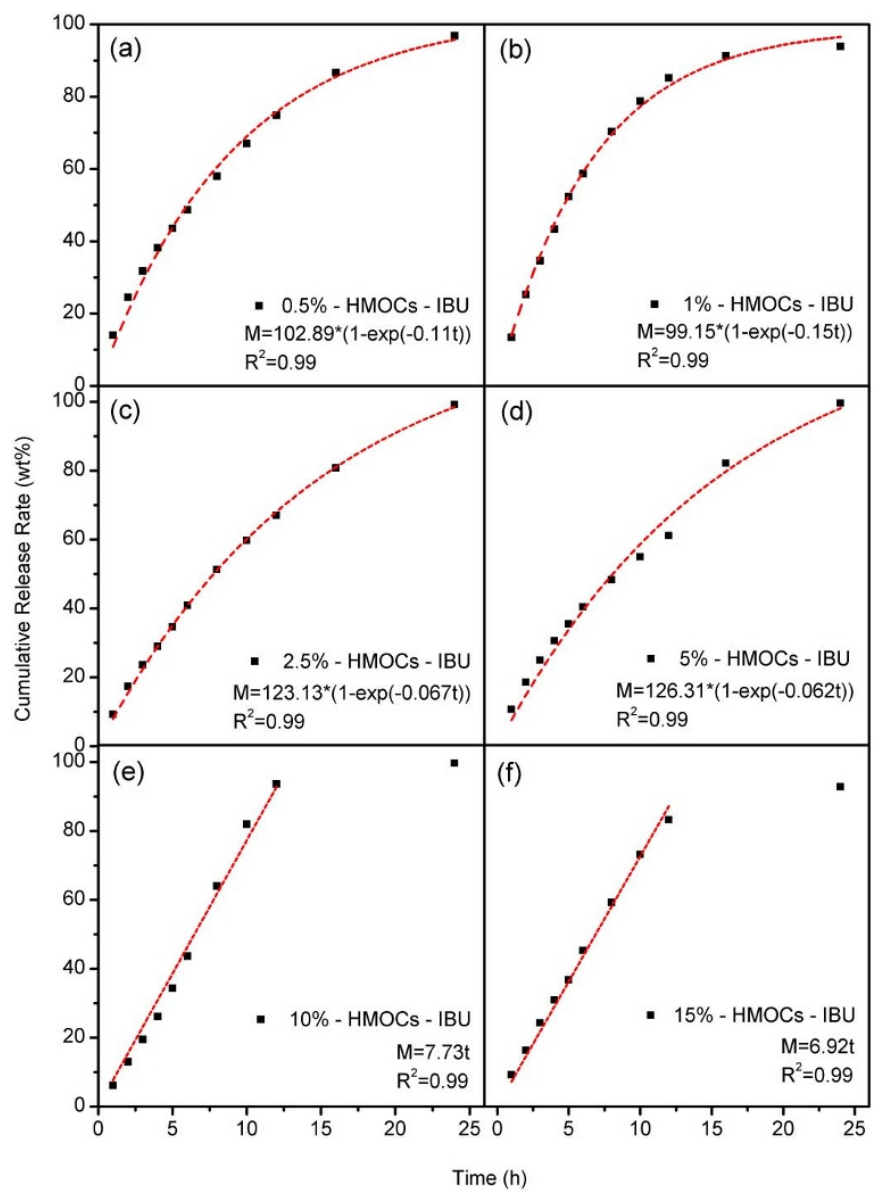

Figure $5 \mid$ Drug release profile of (a) $0.5 \%$ - HMOCs, (b) $1 \%$ - HMOCs, (c) $2.5 \%$ - HMOCs, (d) $5 \%$ - HMOCs, (e) $10 \%$ - HMOCs,

(f) $15 \%$ - HMOCs. Red line is fitting line. structure and their drug release kinetics fit for the first order model. This model indicates that the release mechanism of ibuprofen is mainly controlled by the simple diffusion. HMOCs release the drug at a rate which is proportional to the amount of drug remaining in its interior ${ }^{48}$. However the shells of 10 and $15 \%$ - HMOCs are almost pure microporous and their drug release kinetics fit well for zero order model. As shown in Fig. S40, the molecular size of ibuprofen is up to $10.285 \AA$ which is smaller than the pore size of 10 and $15 \%$ HMOCs.

The micropores are more confined in comparison to mesopores and thus they restrict the free diffusion of drug molecules resulting in a constant drug release rate for $10 \%$ - HMOCs and 15\% - HMOCs with sheer microporous structure. The constant release rate makes the drug amount in blood stable which is an ideal situation for clinical therapy. Compared to the MOFs such as MIL- $53^{49}$ and MIL- $101^{50}$, which have been studied as drug carriers, HMOCs exhibit a higher release rate. But the release behavior of HMOCs is close to that of another microporous organic polymer PAF- ${ }^{51}$ which is the first microporous organic material reported for drug delivery. Although the literature for microporous organic materials for drug delivery is very limited, drug release data of HMOCs still describes their promising prospect as an alternative choice for drug delivery due to their high drug loading efficiency and controlled drug release through adjustable micropore size.

The HMOCs have uniform hollow cores, which can be used as nanoscale reactors to change the composition within the hollow core. For example, herein we have demonstrated it through the synthesis of $5 \mathrm{~nm} \mathrm{Fe}{ }_{3} \mathrm{O}_{4}$ magnetic NPs via $10 \%$ - HMOCs - $2.5 \mathrm{ml}$ as shown in Fig. 6a. $\mathrm{Fe}_{3} \mathrm{O}_{4}$ NPs were formed through co-precipitation of iron precursors $\left(\mathrm{FeCl}_{2}\right.$ and $\left.\mathrm{FeCl}_{3}\right)$, absorbed within the restricted space of hollow cores, using ammonia as precipitator and without using any other external stabilizer. The superparamagnetic properties of $10 \%$ - HMOCs - $2.5 \mathrm{ml}-\mathrm{Fe}_{3} \mathrm{O}_{4} \mathrm{NPs}$, as shown in the Fig. 6b, also prove the existence of $\mathrm{Fe}_{3} \mathrm{O}_{4}$ nanoparticles inside the $\mathrm{HMOCs}^{52}$. After soaking in PBS for 2 days, the magnetic properties of $\mathrm{Fe}_{3} \mathrm{O}_{4}$ nanoparticles loaded HMOCs were maintained, which indicates the confinement of $\mathrm{Fe}_{3} \mathrm{O}_{4}$ nanoparticles within HMOCs cavities. It also implies that the HMOCs- $\mathrm{Fe}_{3} \mathrm{O}_{4} \mathrm{NPs}$ can retain magnetic properties in body fluid. Interestingly, $10 \%$ - HMOCs - $2.5 \mathrm{ml}-\mathrm{Fe}_{3} \mathrm{O}_{4} \mathrm{NPs}$ also showed high drug uptake (2.04 of ibuprofen/g) and zero order kinetic model for drug release (Fig. S41), similar to 10\% - HMOCs - $2.5 \mathrm{ml}$ (2.06 of ibuprofen/g, Fig. S42). The super-paramagnetic properties of $10 \%$ - HMOCs- 2.5 ml- $\mathrm{Fe}_{3} \mathrm{O}_{4}$ NPs were also maintained after drug loading, promising their potential application in the development of magnetically controlled drug delivery systems.

In summary, we have demonstrated the synthesis of a family of hollow microporous organic capsules (HMOCs). Successful efforts
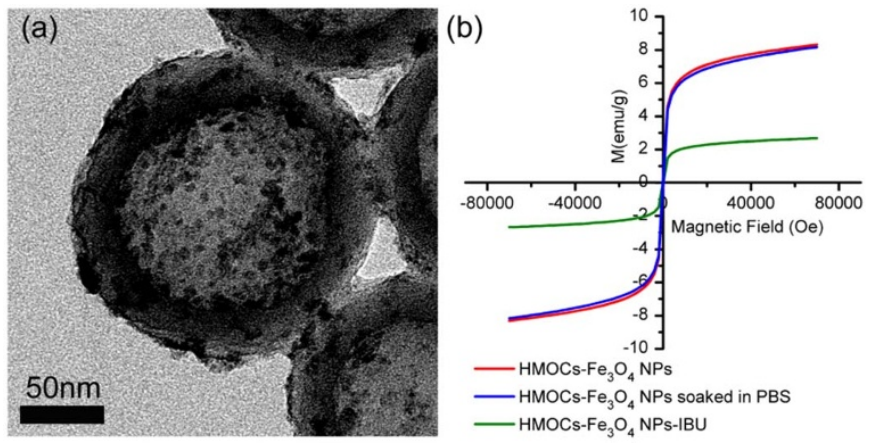

Figure 6 (a) TEM image of $10 \%$ - HMOCs - $2.5 \mathrm{ml} \mathrm{-} \mathrm{Fe}_{3} \mathrm{O}_{4} \mathrm{NPs}$;(b) $300 \mathrm{~K}$ magnetization isotherms of $10 \%$ - HMOCs - $2.5 \mathrm{ml}-\mathrm{Fe}_{3} \mathrm{O}_{4} \mathrm{NPs}$, (red line) $10 \%$ - HMOCs - $2.5 \mathrm{ml}$ - $\mathrm{Fe}_{3} \mathrm{O}_{4} \mathrm{NPs}$ after soaked in PBS for $48 \mathrm{~h}$ (blue line) and 10\% - HMOCs - $2.5 \mathrm{ml}-\mathrm{Fe}_{3} \mathrm{O}_{4}$ NPs loaded with drug (green line). 
were made to precisely control the size of hollow cavities, shell thickness as well as the porous shell structure of these HMOCs. The importance of microporous structure in a new dimension has been demonstrated. Moreover, the multifunctional HMOCs possessing zero order drug release kinetics, fluorescence and super-paramagnetic properties, indicate their attractive applications in medical field. The development of synthetic routes to nanostructured MOPs may receive more scientific attention due to their attractive applications in heterogeneous catalysis and separation technologies.

\section{Methods}

Preparation of hollow microporous hollow capsules (HMOCs). The synthesis protocols for silica nanoparticles are based on the Stöber method. The detailed synthesis procedures for HMOCs are given in the Supplementary information.

Utilization of HMOCs as Confined Microreactors. The HMOCs were dispersed in $0.4 \mathrm{M} \mathrm{FeCl}_{3}$ and $0.2 \mathrm{M} \mathrm{FeCl}_{2}$ aqueous solution. These HMOCs were separated by centrifugation and redispersed in toluene and then mixed with ammonia water, to obtain nanoscale magnetic particles inside the hollow cavity.

Drug loading and release. A typical procedure for the loading of ibuprofen in HMOCs is as follows: $150 \mathrm{mg}$ of HMOCs were suspended in $5 \mathrm{ml}$ of $90 \mathrm{mg} / \mathrm{ml}$ ibuprofen solution in hexane under stirring for $96 \mathrm{~h}$ in a closed container to avoid the evaporation of hexane. The drug-loaded sample was then separated from the solution by vacuum filtration, washed with hexane, and dried at room temperature. The drugloaded samples $(200 \mathrm{mg}$ ) were then transferred to semipermeable bag, and the drug release rate was determined by soaking the drug-loaded samples in $100 \mathrm{ml}$ of simulated body fluid ( $\mathrm{PBS}, \mathrm{pH}=7.4$, buffer solution, $37^{\circ} \mathrm{C}$ ) at pre-determined time intervals, $3 \mathrm{ml}$ samples were withdrawn periodically for analysis and the remaining suspension replenished with an equal volume i.e., $3 \mathrm{~mL}$ of PBS immediately. Samples were analyzed for ibuprofen content at $263 \mathrm{~nm}$ using UV-Vis spectrophometer.

1. Furukawa, H. \& Yaghi, O. M. Storage of Hydrogen, Methane, and Carbon Dioxide in Highly Porous Covalent Organic Frameworks for Clean Energy Applications. J. Am. Chem. Soc. 131, 8875-8883 (2009).

2. McKeown, N. B. et al. Towards Polymer-Based Hydrogen Storage Materials: Engineering Ultramicroporous Cavities within Polymers of Intrinsic Microporosity. Angew. Chem., Int. Ed. 118, 1836-1839 (2006).

3. Wood, C. D. et al. Microporous organic polymers for methane storage. $A d v$. Mater. 20, 1916-1921 (2008)

4. Li, B., Huang, X., Liang, L. \& Tan, B. Synthesis of uniform microporous polymer nanoparticles and their applications for hydrogen storage. J. Mater. Chem. 20, 7444-7450 (2010).

5. Holst, J. R. \& Cooper, A. I. Ultrahigh Surface Area in Porous Solids. Adv. Mater. 22, 5212-5216 (2010).

6. McKeown, N. B. \& Budd, P. M. Polymers of intrinsic microporosity (PIMs): organic materials for membrane separations, heterogeneous catalysis and hydrogen storage. Chem. Soc. Rev. 35, 675-683 (2006).

7. Li, B., Su, F., Luo, H.-K., Liang, L. \& Tan, B. Hypercrosslinked microporous polymer networks for effective removal of toxic metal ions from water. Microporous Mesoporous Mater. 138, 207-214 (2011).

8. Du, X. et al. Troger's base-functionalised organic nanoporous polymer for heterogeneous catalysis. Chem. Commun. 46, 970-972 (2010).

9. Dang, D., Wu, P., He, C., Xie, Z. \& Duan, C. Homochiral Metal-Organic Frameworks for Heterogeneous Asymmetric Catalysis. J. Am. Chem. Soc. 132, 14321-14323 (2010).

10. Li, B. et al. Highly Dispersed Pd Catalyst Locked in Knitting Aryl Network Polymers for Suzuki-Miyaura Coupling Reactions of Aryl Chlorides in Aqueous Media. Adv. Mater. 24, 3390-3395 (2012).

11. Jiang, J. X. \& Cooper, A. Microporous Organic Polymers: Design, Synthesis, and Function. Vol. 293, (Ed.: Schröder, M.), Springer Berlin/Heidelberg, 2010, pp. 1. (2010).

12. Cote, A. P. et al. Porous, crystalline, covalent organic frameworks. Science $\mathbf{3 1 0}$, 1166-1170 (2005).

13. El-Kaderi, H. M. et al. Designed Synthesis of 3D Covalent Organic Frameworks. Science 316, 268-272 (2007).

14. Germain, J., Frechet, J. M. J. \& Svec, F. Nanoporous, hypercrosslinked polypyrroles: effect of crosslinking moiety on pore size and selective gas adsorption. Chem. Commun. 1526-1528 (2009).

15. Germain, J., Frechet, J. M. J. \& Svec, F. Hypercrosslinked polyanilines with nanoporous structure and high surface area: potential adsorbents for hydrogen storage. J. Mater. Chem. 17, 4989-4997 (2007).

16. Tsyurupa, M. P. \& Davankov, V. A. Porous structure of hypercrosslinked polystyrene: State-of-the-art mini-review. React. Funct. Polym. 66, 768-779 (2006).

17. Li, B. et al. A New Strategy to Microporous Polymers: Knitting Rigid Aromatic Building Blocks by External Cross-Linker. Macromolecules 44, 2410-2414 (2011).
18. Budd, P. M. et al. Polymers of intrinsic microporosity (PIMs): robust, solution processable, organic nanoporous materials. Chem. Commun. 230-231 (2004).

19. Jiang, J.-X. et al. Conjugated Microporous Poly(aryleneethynylene) Networks. Angew. Chem. Int. Ed. 47, 1167 (2008).

20. Jiang, J. X et al. Synthetic Control of the Pore Dimension and Surface Area in Conjugated Microporous Polymer and Copolymer Networks. J. Am. Chem. Soc 130, 7710-7720 (2008)

21. Dawson, R., Cooper, A. I. \& Adams, D. J. Nanoporous organic polymer networks. Prog. Polym. Sci. 37, 530-563 (2012).

22. Yuan, S. W. et al. Microporous polyphenylenes with tunable pore size for hydrogen storage. Chem. Commun. 46, 4547-4549 (2010).

23. Kuhn, P., Antonietti, M. \& Thomas, A. Porous, Covalent Triazine-Based Frameworks Prepared by Ionothermal Synthesis. Angew. Chem. Int. Ed. 47, 3450-3453 (2008).

24. Weber, J., Antonietti, M. \& Thomas, A. Microporous networks of highperformance polymers: Elastic deformations and gas sorption properties. Macromolecules 41, 2880-2885 (2008).

25. Pandey, P. et al. Imine-Linked Microporous Polymer Organic Frameworks. Chem. Mater. 22, 4974-4979 (2010).

26. Uribe-Romo, F. J. et al. A Crystalline Imine-Linked 3-D Porous Covalent Organic Framework. J. Am. Chem. Soc. 131, 4570-4571 (2009).

27. Holst, J. R., Stöckel, E., Adams, D. J. \& Cooper, A. I. High Surface Area Networks from Tetrahedral Monomers: Metal-Catalyzed Coupling, Thermal Polymerization, and "Click" Chemistry. Macromolecules 43, 8531-8538 (2010).

28. Pandey, P. et al. A "click-based" porous organic polymer from tetrahedral building blocks. J. Mater. Chem. 21, 1700-1703 (2011).

29. Schmidt, J., Weber, J., Epping, J. D., Antonietti, M. \& Thomas, A. Microporous Conjugated Poly(thienylene arylene) Networks. Adv. Mater. 21, 702-705 (2009).

30. Chen, Q. et al. Microporous Polycarbazole with High Specific Surface Area for Gas Storage and Separation. J. Am. Chem. Soc. 134, 6084-6087 (2012).

31. Yang, X. et al. Magnetic microporous polymer nanoparticles. Polym. Chem. 4, 1425-1429 (2013).

32. Chun, J. et al. Tubular-Shape Evolution of Microporous Organic Networks. Chem. Mater. 24, 3458-3463 (2012).

33. Patra, A., Koenen, J.-M. \& Scherf, U. Fluorescent nanoparticles based on a microporous organic polymer network: fabrication and efficient energy transfer to surface-bound dyes. Chem. Commun. 47, 9612 (2011).

34. Jiang, X. \& Brinker, C. J. Aerosol-Assisted Self-Assembly of Single-Crystal Core/ Nanoporous Shell Particles as Model Controlled Release Capsules. J. Am. Chem. Soc. 128, 4512-4513 (2006)

35. Quan, B., Nam, G.-E., Choi, H. J. \& Piao, Y. Synthesis of Monodisperse Hollow Carbon Nanocapsules by Using Protective Silica Shells. Chem-Asian J. 8, 765-760 (2013).

36. Sanlés-Sobrido, M., Pérez-Lorenzo, M., Rodríguez-González, B., Salgueiriño, V. \& Correa-Duarte, M. A. Highly Active Nanoreactors: Nanomaterial Encapsulation Based on Confined Catalysis. Angew. Chem. Int. Ed. 51, 3877-3882 (2012).

37. Arnal, P. M., Comotti, M. \& Schüth, F. High-Temperature-Stable Catalysts by Hollow Sphere Encapsulation. Angew. Chem. Int. Ed. 45, 8224-8227 (2006).

38. Shi, J., Li, X., Wang, Q., Zhang, Y. \& Tang, Y. Platinum-encapsulated zeolitically microcapsular catalyst for one-pot dynamic kinetic resolution of phenylethylamine. J. Catal. 291, 87-94 (2012).

39. Fontanals, N. et al. Synthesis of Davankov-Type Hypercrosslinked Resins Using Different Isomer Compositions of Vinylbenzyl Chloride Monomer, and Application in the Solid-Phase Extraction of Polar Compounds. J. Polym. Sci., Part A: Polym. Chem. 43, 1718-1728 (2005).

40. Ahn, J. H. et al. Rapid Generation and Control of Microporosity, Bimodal Pore Size Distribution, and Surface Area in Davankov-Type Hyper-Cross-Linked Resins. Macromolecules 39, 627-632 (2006)

41. Caruso, F. Modern Techniques for Nano- and Microreactors/-reactions. (Springer, 2010).

42. Wu, D. et al. Nanoporous Polystyrene and Carbon Materials with Core-Shell Nanosphere-Interconnected Network Structure. Macromolecules 44, 5846-5849 (2011).

43. Zhang, K. et al. Monodisperse Silica-Polymer Core-Shell Microspheres via Surface Grafting and Emulsion Polymerization. Macromol. Mater. Eng. 288, 380-385 (2003).

44. Li, B., Gong, R., Luo, Y. \& Tan, B. Tailoring the pore size of hypercrosslinked polymers. Soft Matter 7, 10910-10916 (2011).

45. Harkins, W. D. A General Theory of the Mechanism of Emulsion Polymerization1. J. Am. Chem. Soc. 69, 1428-1444 (1947).

46. Sing, K. S. W. et al. Reporting Physisorption Data for Gas/Solid Systems with Special Reference to the Determination of Surface Area and Porosity. Pure \& Appl. Chem. 57, 603-619 (1985).

47. Chen, L.-T. \& Weiss, L. The Role of the Sinus Wall in the Passage of Erythrocytes Through the Spleen. Blood 41, 529-537 (1973).

48. Costa, P. \& Sousa Lobo, J. M. Modeling and comparison of dissolution profiles. Eur. J. Pharm. Sci. 13, 123-133 (2001).

49. Horcajada, P. et al. Flexible Porous Metal-Organic Frameworks for a Controlled Drug Delivery. J. Am. Chem. Soc. 130, 6774-6780 (2008).

50. Horcajada, P. et al. Metal-Organic Frameworks as Efficient Materials for Drug Delivery. Angew. Chem. Int. Ed. 45, 5974-5978 (2006). 
51. Zhao, H. et al. Targeted synthesis of a 2D ordered porous organic framework for drug release. Chem. Commun. 47, 6389-6391 (2011).

52. Xu, X. \& Asher, S. A. Synthesis and Utilization of Monodisperse Hollow Polymeric Particles in Photonic Crystals. J. Am. Chem. Soc. 126, 7940-7945 (2004).

\section{Acknowledgements}

We thank Analysis and Testing Center, Huazhong University of Science and Technology for their assistance in characterization of materials. This work was financially supported by the program for New Century Excellent Talents in University (NCET-10-0389), the Graduates' Innovation Fund of Huazhong University of Science and Technology (HF-11-07-2013), Program for Changjiang Scholars and Innovative Research Team in University (PCSIRT) and National Natural Science Foundation of China (No. 51173058/ 51273074)

\section{Author contributions}

B.L. and X.Y. synthesized and characterized the HMOCs. X.L. performed the drug release experiments. B.T., B. L. and M. I. M. discussed the results and prepared the manuscript. B.T. conceived the project.

\section{Additional information}

Supplementary information accompanies this paper at http://www.nature.com/ scientificreports

Competing financial interests: The authors declare no competing financial interests. How to cite this article: Li, B.Y., Yang, X.J., Xia, L.L., Majeed, M.I. \& Tan, B. Hollow Microporous Organic Capsules. Sci. Rep. 3, 2128; DOI:10.1038/srep02128 (2013).

(c) (1) (2)(2) This work is licensed under a Creative Commons AttributionNonCommercial-ShareAlike 3.0 Unported license. To view a copy of this license visit http://creativecommons.org/licenses/by-nc-sa/3.0 